\title{
Cerebellar Functional Anatomy: a Didactic Summary Based on Human fMRI Evidence
}

\author{
Xavier Guell ${ }^{1,2} \cdot$ Jeremy Schmahmann ${ }^{1}$ \\ Published online: 9 November 2019 \\ (C) Springer Science+Business Media, LLC, part of Springer Nature 2019
}

\begin{abstract}
The cerebellum is relevant for virtually all aspects of behavior in health and disease. Cerebellar findings are common across all kinds of neuroimaging studies of brain function and dysfunction. A large and expanding body of literature mapping motor and non-motor functions in the healthy human cerebellar cortex using fMRI has served as a tool for interpreting these findings. For example, results of cerebellar atrophy in Alzheimer's disease in caudal aspects of Crus I/II and medial lobule IX can be interpreted by consulting a large number of task, resting-state, and gradient-based reports that describe the functional characteristics of these specific aspects of the cerebellar cortex. Here, we provide a concise summary that outlines organizational principles observed consistently across these studies of normal cerebellar organization. This basic framework may be useful for investigators performing or reading experiments that require a functional interpretation of human cerebellar topography.
\end{abstract}

\section{Introduction}

Numerous studies have mapped motor and non-motor task processes and resting-state networks in the human cerebellar cortex using fMRI [1-12]. This field of research has contributed to the development of modern cerebellar systems neuroscience- the cerebellum is now appreciated as a structure relevant for virtually all aspects of behavior in health and disease (see [13]). This large body of human cerebellar neuroimaging literature has also served as a tool for interpreting the functional significance of cerebellar neuroimaging findings in studies of brain disorders. For example, a study of a neurological condition reporting decreased volume in the intersection between left cerebellar Crus I and Crus II is now informed by a large number of task, resting-state, and gradient-based reports that describe the functional characteristics of this specific aspect of the cerebellar cortex. The objective of

Xavier Guell

xaviergp@mit.edu

Jeremy Schmahmann

jschmahmann@mgh.harvard.edu

1 Department of Neurology, Massachusetts General Hospital, Harvard Medical School, 55 Fruit St., Boston, MA, USA

2 Department of Brain and Cognitive Sciences, McGovern Institute for Brain Research at MIT, Massachusetts Institute of Technology, 43 Vassar St., Cambridge, MA, USA this synopsis is to provide a concise summary that outlines organizational principles observed consistently in functional imaging studies of the human cerebellum. This basic framework may help guide the interpretation of human cerebellar topography in functional and morphometric imaging studies.

\section{Three Functional Subdivisions: Motor, Attentional/Executive (Task-Positive), and Default-Mode (Task-Negative)}

Data-driven analyses of cerebellar fMRI data indicate that (i) motor, (ii) attentional/executive (task-positive), and (iii) default-mode (task-negative) processing are three fundamental poles of cerebellar functional neuroanatomy [5]. Our didactic summary will be based on this division (Fig. 1A). Processes that cannot be clearly classified according to these categories (e.g., emotion processing, or the property of language lateralization) will be discussed separately.

Motor processing refers to regions in the cerebellar cortex that exhibit activation in fMRI task contrasts such as moving $a$ finger following a visual cue minus observing the same cue without performing any movement, or regions in the cerebellar cortex that exhibit functional connectivity to cerebral motor areas such as M1. Attentional/executive processing refers to cerebellar cortical areas that exhibit functional connectivity to cerebral systems relevant for stimulus-driven attention (ventral attention network, salience network), goal-oriented 


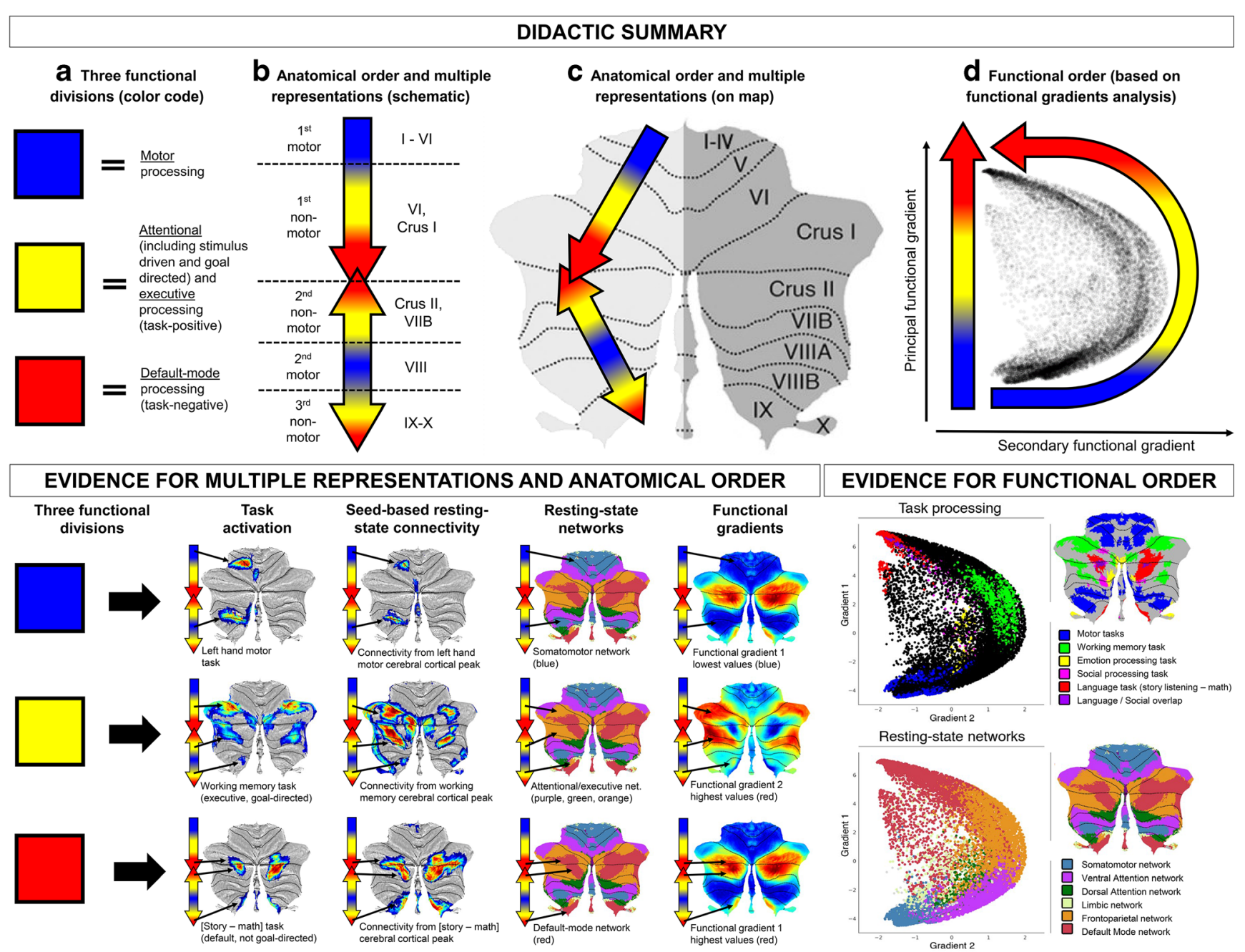

Fig. 1 Didactic summary of cerebellar functional anatomy based on human fMRI evidence. Top row: (A) Data-driven analyses of cerebellar fMRI indicate that motor, attentional/executive (task-positive), and default-mode (task-negative) processing constitute three fundamental poles of cerebellar functional neuroanatomy [5]. Our didactic summary is based on this division. (B, C) Motor processing is represented twice in each cerebellar cortical hemisphere (lobules I-VI; lobule VIII). Nonmotor processes (attentional/executive and default-mode) are represented three times in each cerebellar cortical hemisphere (lobules VI-Crus I; lobules Crus II-VIIB; lobules IX-X). A specific anatomical order from motor (blue), to attentional/executive (yellow), to defaultmode territories (red) is conserved throughout the cerebellar cortex. This specific functional ordering (motor, attentional/executive, defaultmode) propagates from first motor towards first non-motor representation (i.e., from lobules I-VI to Crus I), from second motor towards second non-motor representation (i.e., from lobule VIII to Crus II), and from second motor towards third non-motor representation (i.e., from lobule VIII to IX/X). Of note, first and second non-motor

attention (dorsal attention network), and related executive functions (frontoparietal network). Tasks engaging these systems include contrasts such as working memory 2-back minus 0 -back conditions. Default-mode processing refers to cerebellar cortical areas that exhibit functional connectivity to cerebral default network [14], engagement in inattentive states representations can be contiguous (as in the case of default-mode processing shown in red, see overlapping red arrows in Crus I/II) or separate (as in the case of attentional/executive processing shown in yellow). (D) The principal axis of macroscale functional organization in the cerebellar cortex progresses from motor, to attentional/executive, to default-mode processing. This progression is captured in the anatomical order of cerebellar functional territories as shown in (B, C), and also revealed by a data-driven analysis of functional gradients in the cerebellar cortex based on resting-state functional connectivity between cerebellar cortical areas (Guell et al. [5]; functional gradient 1 shown in the $\mathrm{y}$ axis progresses from motor, to attentional/executive, to defaultmode processing). Functional gradient 2 captures a smaller portion of data variability and reveals that a secondary axis in cerebellar macroscale functional neuroanatomy isolates attentional/executive processing. Bottom row: evidence supporting the organizational principles presented in the top row: task activation [2], seed-based resting-state connectivity [2], cerebellar mapping of cerebral cortical resting-state networks [1], and functional gradients [5]

such as mind wandering, and deactivation during highly attention-demanding processes. Task contrasts engaging this system include listening to a story minus doing math, where attentional demands are subtracted in the control condition.

A division of cognition based on default-mode versus attentional/executive processing is supported across multiple 
human and animal studies of brain physiology. Default-mode and attentional/executive territories are anti-correlated at rest [15], dissociated in inattentive as opposed to vigilant brain state activity $[16,17]$, and causally interfere with the activation of each other [18].

\section{Anatomical Order in the Cerebellar Cortex}

These three functional domains are organized anatomically in the cerebellar cortex obeying the following four principles:

(i) Motor processing is represented twice in each cerebellar cortical hemisphere (lobules I-VI; lobule VIII). Nonmotor processes (attentional/executive and default-mode) are represented three times in each cerebellar cortical hemisphere (lobules VI-Crus I; lobules Crus II-VIIB; lobules IX-X). There are thus two motor [19] and three nonmotor [1, 2] representations in cerebellar cortex (Fig. 1B, C).

(ii) A specific anatomical order from (a) motor, to (b) attentional/executive, to (c) default-mode territories is conserved throughout the cerebellar cortex (Fig. 1B, C) (e.g., see $[1,5])$. This organization is similar to what previous studies have reported in the cerebral cortex (in the case of the cerebral cortex, a basic conserved organization throughout many cortical regions progresses from primary motor/auditory/visual, to attentional/executive, to default-mode territories; see [20-22]).

(iii) The two principles stated so far result in the propagation of a specific functional ordering (motor, attentional/executive, default-mode) from first motor towards first non-motor representation (i.e., from lobules I-VI to Crus I), from second motor towards second non-motor representation (i.e., from lobule VIII to Crus II), and from second motor towards third non-motor representation (i.e., from lobule VIII to IX/X) (see the three arrows in Fig. 1C).

(iv) This organization results in the anatomical peculiarity that Crus I-Crus II intersection is the intersection of first default-mode representation and second default-mode representation. In this way, first and second non-motor representations can be contiguous (as in the case of default-mode processing) or separate (as in the case of attentional/executive processing) (see overlapping arrows in Crus I/II in Fig. 1C).

\section{Functional Order in the Cerebellar Cortex}

The principal axis of macroscale functional organization in the cerebellar cortex progresses from motor, to attentional/executive, to default-mode processing (Fig. 1D). This progression is captured in the anatomical order of cerebellar functional territories as discussed in the previous section (principle ii; Fig. 1B, C), and also revealed by a data-driven analysis of functional gradients in the cerebellar cortex based on restingstate functional connectivity between cerebellar cortical areas (Guell et al. [5]; functional gradient 1 progresses from motor, to attentional/executive, to default-mode processing). Functional gradient 1 captures the highest portion of variability in cerebellar cortical resting-state functional connectivity patterns. Functional gradient 2 captures a smaller portion of data variability and reveals that a secondary axis in cerebellar macroscale functional neuroanatomy isolates attentional/ executive processing. These two dimensions (functional gradients 1 and 2) provide an alternative functional rather than anatomical space for the visualization of the results of cerebellar neuroimaging [23].

\section{Special Cases: Emotion, Vestibular, Language, and Social Processing}

Some functional domains require special consideration because of peculiarities of their anatomical or functional distribution in cerebellar cortex that are not captured by the summary provided in the previous sections.

Emotion processing follows the principle of a triple representation in the cerebellar cortex [2], but it is located centrally along functional gradients 1 and 2. It is therefore not possible to classify emotion processing wholly within default-mode (high gradient 1 values), motor (low gradient 1 values), and attentional/executive (high gradient 2 values) divisions [5]. Emotion processing also shows a tendency to engage cerebellar vermis, consistent with the theory of a vermal location of emotion processing in the cerebellum [2, 24, 25]. Within the framework presented here, Crus I/II vermal engagement in emotion processing is viewed as a first and contiguous second representation, and lobule IX/X emotion processing activation corresponds to the third representation [2].

Vestibular functions are in many cases difficult to investigate within the constraints of fMRI experimental designs. Physiology and anatomy investigations in animals and clinical studies in humans underscore the engagement of cerebellar vermis (predominantly vermal lobules V-VII, named oculomotor vermis) as well as lobules IX and $\mathrm{X}$ in vestibular control $[26,27]$. Human fMRI experiments map ocular movements to these regions $[3,28]$. It is possible that there is an overlap between oculomotor control and visual attention in the cerebellum [3, 29], as well as links between vestibular and emotion processing that both engage vermal aspects of the cerebellar cortex [30, 31].

Language processing in the cerebellar cortex has a wellestablished predominant right lateralization that mirrors the predominant left lateralization of language processing in the cerebral cortex. This distribution follows the logic of 
anatomical connections that link the majority of cerebellar cortex to the contralateral cerebral hemisphere $[32,33]$.

Social cognition processing in the cerebellum overlaps with default-mode processing territories [2,34], reflecting an engagement of default-mode network in processes that are relevant for social cognition $[14,35]$. Social cognition also extends diffusely along functional gradient 1 , resonating with the understanding that this multimodal function engages multiple levels of information processing along the principal dimensions of cerebellar functional neuroanatomy, without exclusive localization at any of its poles (default-mode, attentional/executive, or motor) [5].

\section{Conclusion}

Here, we have outlined general principles that remain broadly observable across a large and growing body of literature describing the functional organization of human cerebellar cortex [1-3, $5-12,36]$. These principles are based on the notion of multiple areas of motor and non-motor representations, and a specific ordering of functional domains (motor, attentional/executive, and default-mode) that together define the position of, and relationship between, each functional territory in cerebellar macroscale functional anatomy. Structural and functional neuroimaging experiments often reveal findings in the cerebellum that are relevant for many aspects of clinical and fundamental neuroscience beyond the field of imaging. The summary presented here may be useful for investigators performing or reading experiments that require a functional interpretation of human cerebellar topography. For a more nuanced understanding of cerebellar functional neuroanatomy that goes beyond the basic framework presented here, we encourage the readers to consult the references included in this article.

\section{Compliance with Ethical Standards}

Conflict of Interest The authors declare that they have no conflict of interest.

\section{References}

1. Buckner RL, Krienen FM, Castellanos A, Diaz JC, Yeo BTT. The organization of the human cerebellum estimated by intrinsic functional connectivity. J Neurophysiol. 2011;106(5):2322-45.

2. Guell X, Gabrieli JDE, Schmahmann JD. Triple representation of language, working memory, social and emotion processing in the cerebellum: convergent evidence from task and seed-based resting-state fMRI analyses in a single large cohort. Neuroimage. 2018;172:437-49.

3. King M, Hernandez-Castillo CR, Poldrack RA, Ivry RB, Diedrichsen J. Functional boundaries in the human cerebellum revealed by a multidomain task battery. Nat Neurosci. 2019;22:1371-8.
4. Brissenden JA, Levin EJ, Osher DE, Halko MA, Somers DC. Functional evidence for a cerebellar node of the dorsal attention network. J Neurosci. 2016;36(22):6083-96.

5. Guell X, Schmahmann J, Gabrieli J, Ghosh S. Functional gradients of the cerebellum. Elife. 2018;7:e36652.

6. Stoodley CJ, Valera EM, Schmahmann JD. An fMRI study of intraindividual functional topography in the human cerebellum. Behav Neurol. 2010;23(1-2):65-79.

7. Keren-Happuch E, Chen SHA, Ho MHR, Desmond JE. A metaanalysis of cerebellar contributions to higher cognition from PET and fMRI studies. Hum Brain Mapp. 2014;35(2):593-615.

8. Stoodley CJ, Schmahmann JD. Functional topography in the human cerebellum: a meta-analysis of neuroimaging studies. Neuroimage. 2009;44(2):489-501.

9. Stoodley CJ, Valera EM, Schmahmann JD. Functional topography of the cerebellum for motor and cognitive tasks: an fMRI study. Neuroimage. 2012;59(2):1560-70.

10. Habas C, Kamdar N, Nguyen D, Prater K, Beckmann CF, Menon V, et al. Distinct cerebellar contributions to intrinsic connectivity networks. J Neurosci. 2009;29(26):8586-94.

11. O'Reilly JX, Beckmann CF, Tomassini V, Ramnani N, JohansenBerg H. Distinct and overlapping functional zones in the cerebellum defined by resting state functional connectivity. Cereb Cortex. 2010;20(4):953-65.

12. Marek S, Siegel JS, Gordon EM, Raut RV, Gratton C, Newbold DJ, et al. Spatial and temporal organization of the individual cerebellum. Neuron. 2018;100:977-93.

13. Schmahmann J, Guell X, Stoodley C, Halko M. The theory and neuroscience of cerebellar cognition. Annu Rev Neurosci. 2019;42: 337-64.

14. Buckner RL, Andrews-Hanna JR, Schacter DL. The brain's default network: anatomy, function, and relevance to disease. Ann N Y Acad Sci. 2008;1124:1-38.

15. Fox MD, Snyder AZ, Vincent JL, Corbetta M, Van Essen DC, Raichle ME. The human brain is intrinsically organized into dynamic, anticorrelated functional networks. Proc Natl Acad Sci. 2005;102(27):9673-8.

16. Hayden BY, Smith DV, Platt ML. Electrophysiological correlates of default-mode processing in macaque posterior cingulate cortex. Proc Natl Acad Sci. 2009;106(14):5948-53.

17. Barch DM, Burgess GC, Harms MP, Petersen SE, Schlaggar BL, Corbetta M, et al. Function in the human connectome: task-fMRI and individual differences in behavior. Neuroimage. 2013;80:169-89.

18. Chen AC, Oathes DJ, Chang C, Bradley T, Zhou Z-W, Williams LM, et al. Causal interactions between fronto-parietal central executive and default-mode networks in humans. Proc Natl Acad Sci. 2013;110(49):19944-9.

19. Snider R, Eldred E. Cerebrocerebellar relationships in the monkey. J Neurophysiol. 1952;15(1):27-40.

20. Braga RM, Buckner RL. Parallel interdigitated distributed networks within the individual estimated by intrinsic functional connectivity. Neuron. 2017;95(2):457-471.e5.

21. Margulies DS, Ghosh SS, Goulas A, Falkiewicz M, Huntenburg JM, Langs G, et al. Situating the default-mode network along a principal gradient of macroscale cortical organization. Proc Natl Acad Sci. 2016;113(44):12574-9.

22. Yeo B, Krienen FM, Sepulcre J, Sabuncu MR, Lashkari D, Hollinshead M, et al. The organization of the human cerebral cortex estimated by intrinsic functional connectivity. J Neurophysiol. 2011;106(3):1125-65.

23. Guell X, Goncalves M, Kaczmarzyk J, Gabrieli J, Schmahmann J, Ghosh S. LittleBrain: a gradient-based tool for the topographical interpretation of cerebellar neuroimaging findings. PLoS One. 2019;14(1):e0210028.

24. Schmahmann J. An emerging concept: The cerebellar contribution to higher function. Arch Neurol. 1991;48(11):1178-87. 
25. Schmahmann JD, Sherman JC. The cerebellar cognitive affective syndrome. Brain. 1998;121(4):561-79.

26. Goldberg JM, Wilson VJ, Cullen KE, Angelaki DE, Broussard DM, Buttner-Ennever J, et al. The vestibular system: a sixth sense. 1st ed. The Vestibular System: A Sixth Sense. Oxford Scholarship Online; 2012

27. Manto M, Mariën P. Schmahmann's syndrome - identification of the third cornerstone of clinical ataxiology. Cerebellum Ataxias. $2015 ; 2: 2$.

28. Voogd J, Schraa-Tam CKL, Van Der Geest JN, De Zeeuw CI. Visuomotor cerebellum in human and nonhuman primates. Cerebellum. 2012;11(2):392-410.

29. van Es DM, van der Zwaag W, Knapen T. Topographic maps of visual space in the human cerebellum. Curr Biol. 2019;29(10): 1689-1694.e3.

30. Levinson HN. The cerebellar-vestibular predisposition to anxiety disorders. Percept Mot Skills. 1989;68(1):323-38.

31. Schmahmann JD, Weilburg JB, Sherman JC. The neuropsychiatry of the cerebellum - Insights from the clinic. Cerebellum. 2007;6(3): 254-67.
32. Gelinas JN, Fitzpatrick KPV, Kim HC, Bjornson BH. Cerebellar language mapping and cerebral language dominance in pediatric epilepsy surgery patients. NeuroImage Clin. 2014;6:296-306.

33. Marien P, Engelborghs S, Fabbro F, De Deyn PP. The lateralized linguistic cerebellum: a review and a new hypothesis. Brain Lang. 2001;79(3):580-600.

34. Van Overwalle F, Baetens K, Mariën P, Vandekerckhove M. Cerebellar areas dedicated to social cognition? A comparison of meta-analytic and connectivity results. Soc Neurosci. 2015;10(4):337-44.

35. Mars RB, Neubert F-X, Noonan MP, Sallet J, Toni I, Rushworth MFS. On the relationship between the "default mode network" and the "social brain.". Front Hum Neurosci. 2012;6:189.

36. Van Overwalle F, Baetens K, Mariën P, Vandekerckhove M. Social cognition and the cerebellum: a meta-analysis of over $350 \mathrm{fMRI}$ studies. Neuroimage. 2014;86:554-72.

Publisher's Note Springer Nature remains neutral with regard to jurisdictional claims in published maps and institutional affiliations. 